\title{
Bilateral Primary Ovarian Clear Cell Carcinoma in an Iranian Woman: a Case Report
}

\author{
Fatemeh Samiee-Rad ${ }^{1}$ (D) Mahdi Ghaebi ${ }^{2}$ (D) Arezoo Bajelan $^{2}$ (B) \\ Received: 23 May 2020 / Accepted: 15 September 2020 \\ (C) Indian Association of Surgical Oncology 2020
}

\section{Background}

Ovarian neoplasms have one of the highest mortality rates among all gynecologic cancers. Among Iranian women, 5 -year survival of the patients with ovarian cancer is approximately $60 \%$ [1]. Ovarian clear cell carcinoma (OCCC) is a subtype of epithelial ovarian carcinomas (EOCs), which is characterized by clear cells in solid, tubular or papillary patterns, and also hobnail cells lining tubules and cysts [2].

Ovarian and renal clear cell carcinomas have similar pathological properties, and both have cells with clear cytoplasm, due to glycogen accumulation in the cytoplasm. Therefore, the possibility of renal origin of ovarian clear cell tumors, especially in bilateral cases, should be investigated [3].

The prevalence of OCCC in American population is estimated to be around 5 to $10 \%$ of all EOCs, and it has a higher prevalence in Asian countries, especially in East Asia [4]. It is believed that genetics has a considerable role in developing OCCC. The genetic profile of OCCC demonstrates frequent mutations in ARID1A (AT-rich interaction domain 1A), PIK3CA (phosphatidylinositol-4, 5-bisphosphate 3-kinase catalytic sub-unit alpha), MET (mesenchymal-epithelial transition) amplification, and P53 [4].

Fatemeh Samiee-Rad

fsamieerad@gmail.com

Mahdi Ghaebi

mehdighaebi1992@gmail.com

Arezoo Bajelan

aarezoo.b@gmail.com

1 Faculty of Medical School, Qazvin University of Medical Sciences, Bahonar St, Qazvin, Iran

2 General Physician, Qazvin University of Medical Sciences, Bahonar St, Qazvin, Iran
The characteristics and clinical features of $\mathrm{OCCC}$ are different from other EOCs. OCCC is highly prone to recurrence and shows low chemosensitivity to platinum-based front-line chemotherapy [5]. It usually develops after the sixth decade of life, with symptoms such as palpable pelvic mass, abdominal pain, gastrointestinal symptoms, and abdominal distension [6]. Aging, nulliparity, early menarche or late menopause, genetics, and endometriosis are known to be risk factors for developing ovarian clear cell carcinoma [7]. Factors such as multiparity, oral contraceptive use, and lactation are believed to decrease the risk of OCCC [8]. OCCC is found both unilaterally and bilaterally. Only $10 \%$ of all cases and $4 \%$ of stage I cases are bilateral [9].

A systematic review study has confirmed that there is an increased risk of ovarian cancer, especially endometrioid carcinoma and clear cell carcinoma, in patients with a positive history of endometriosis [10].

OCCC usually spreads to the peritoneum and omentum by local extension, lymphatic invasion, intraperitoneal implantation, hematogenous dissemination, or trans-diaphragmatic passage. High stages of the disease can be found with metastasis to bone, lungs, liver, and skin [11]. The primary workup should include an ultrasound and/or abdominopelvic computerized tomography (CT) scan/magnetic resonance imaging $(M R I)$, appropriate laboratory studies, tumor markers, serous effusion cytopathology analysis, and histopathology study of resected specimen [12].

Primary OCCC is a less common event. Most of the time, it is found in nulliparous postmenopausal women associated with past medical history of endometriosis and present after the sixth decade of life. However, based on our literature review, occurrence of bilateral primary OCCC in multiparous perimenopausal patient before 50 years old without any association with endometriosis is very rare [7-9].

Herein, we report a case of bilateral primary ovarian clear cell carcinoma in an Iranian woman and summarize radiologic and histopathology findings. 